Final Report on Phase II DoE SBIR grant DE-FG02-08 ER85206

Topic 50b: RADIO FREQUENCY POWER FOR LINEAR ACCELERATORS

HIGH-POWER MICROWAVE SWITCH EMPLOYING ELECTRON BEAM TRIGGERING

Omega-P, Inc.

291 Whitney Avenue - Suite 401

New Haven, CT 06511

\title{
HIGH-POWER MICROWAVE SWITCH EMPLOYING ELECTRON BEAM TRIGGERING
}

\author{
J. L. Hirshfield, Principal Investigator \\ tel: (203) 789-1164, e-mail: jay@omega-p.com
}

Final Report on Phase II DoE SBIR grant DE-FG02-08 ER85206

Topic 50b: RADIO FREQUENCY POWER FOR LINEAR ACCELERATORS

\begin{abstract}
A high-power active microwave pulse compressor is described that modulates the quality factor $Q$ of the energy storage cavity by a new means involving mode conversion controlled by a triggered electron-beam discharge through a switch cavity. The electron beam is emitted from a diamond-coated molybdenum cathode. This report describes the principle of operation, the design of the switch, the configuration used for the test, and the experimental results. The pulse compressor produced output pulses with 140-165 MW peak power, power gain of 16-20, and pulse duration of 16-20 ns at a frequency of $11.43 \mathrm{GHz}$.
\end{abstract}

\section{TABLE OF CONTENTS}

I. Introduction 2

II. Technical approach

III. Conclusions and acknowledgments

IV. References

Work on this project was carried out by O.A. Ivanov, M.A. Lobaev, A.L. Vikharev, A.M. Gorbachev, and V.A. Isaev, of Omega-P, Inc. and Institute of Applied Physics, RAS; S.H. Gold ${ }^{4}$ and A.K. Kinkead, of US Naval Research Laboratory; and J. L. Hirshfield of Omega-P, Inc. Dr. J.L. Hirshfield, serving as Principal Investigator, edited this report.

September 19, 2012 
Final Report on Phase II DoE SBIR grant DE-FG02-08 ER85206

Topic 50b: RADIO FREQUENCY POWER FOR LINEAR ACCELERATORS

\section{HIGH-POWER MICROWAVE SWITCH EMPLOYING ELECTRON BEAM TRIGGERING}

\section{INTRODUCTION}

A proposed future $\mathrm{TeV}$ electron-positron linear collider operating at $\mathrm{X}$-band, and a test facility for its components, will require high peak microwave powers of hundreds of megawatts in pulses lasting hundreds of nanoseconds. One method to provide such pulses is through the use of microwave pulse compressors to multiply the power produced by high-power klystrons producing multi-microsecond pulse widths. Such compressors can be passive, employing phase switching to discharge microwave energy storage cavities, such as SLED 2 [1], or active [2], in which a triggered increase in the output coupling coefficient of the energy storage cavities is used to extract a high power microwave pulse. Active microwave pulse compressors are of interest because they are capable of both higher compression ratios and higher efficiencies than passive compressors. Up until now, the best results in active pulse compression were obtained in a series of experiments where the authors investigated active pulse compressors that used a plasma discharge inside gas-filled dielectric tubes located within switch cavities to shift the switch cavities into resonance, and thus to lower the $\mathrm{Q}$ of the energy storage cavities. Those experiments produced compressed pulses of 50-70 MW peak power and 40-70 ns duration, with peak power gains of 7:1-11:1 and efficiencies in the range of 50\%-63\% [2]. The results were limited by the appearance of a multipactor discharge on the surface of the dielectric tubes and by self-breakdown of the low-pressure gas contained in the tubes.

These experiments suggested that higher power operation should be possible if the switching elements were removed from the switch cavities by replacing the intra-cavity plasma discharge by an externally injected electron beam. This concept was first successfully tested in low power experiments $[3,4]$. In this paper we report the first high power test of a novel twochannel active microwave pulse compressor with switches based on the new concept of triggering using an electron beam discharge. This paper describes the design of the switch, the principle of operation of the compressor, and the experimental configuration tested using an 11.43-GHz magnicon amplifier [5, 6] as the high-power source. The experiments were carried out at drive powers of up to $9 \mathrm{MW}$ in a $\sim 1.1 \mu$ s pulse and produced output pulses with peak powers of 140-165 MW, power gains of 16-20, and pulse durations of 16-20 ns. These results 
Final Report on Phase II DoE SBIR grant DE-FG02-08 ER85206

Topic 50b: RADIO FREQUENCY POWER FOR LINEAR ACCELERATORS

\section{HIGH-POWER MICROWAVE SWITCH EMPLOYING ELECTRON BEAM TRIGGERING}

suggest the feasibility of employing active pulse compressors in a future $\mathrm{TeV}$ electron-positron collider.

\section{TECHNICAL APPROACH}

In the new compressor, microwave energy is stored in the high-Q $\mathrm{TE}_{02}$ mode of two parallel-fed transmission line cavities, and is switched out from them in the $\mathrm{TE}_{01}$ mode. The switches are based on a rapid increase in the $\mathrm{TE}_{02} \rightarrow \mathrm{TE}_{01}$ mode conversion coefficient when an electron beam is injected into the switch cavities. The compressor comprises two identical single-channel compressors that are fed by a 3-dB hybrid coupler, thus isolating the microwave generator from the power reflected during energy storage without requiring a high power, vacuum compatible circulator. The design and operating principles of a single compressor channel are illustrated in Fig. 1(a). Microwave energy is fed to the input of the compressor channel in the $\mathrm{TE}_{01}{ }^{\mathrm{O}}$ mode of a circular waveguide. The compressor channel consists of a coupling waveguide (1) that is cut off to the $\mathrm{TE}_{02}$ mode, an input horn (2), a section of cylindrical waveguide (3), and the switch region (4-9). The input horn, cylindrical waveguide, and switch region form a storage cavity for the $\mathrm{TE}_{02}$ mode. In the regime of energy storage, a small fraction $(\sim 1-3 \%)$ of the $\mathrm{TE}_{01}$ mode supplied by the microwave source via the input waveguide is converted into the $\mathrm{TE}_{02}$ mode at the conical taper (4). The $\mathrm{TE}_{02}$ mode is completely reflected from input horn (2). As a result, the microwave energy builds up in the cavity in the $\mathrm{TE}_{02}$ mode.

The switch region consists of a conical taper (4), in which the $\mathrm{TE}_{02}$ and $\mathrm{TE}_{01}$ modes are coupled to each other, a waveguide section (5) of adjustable length, and a $\mathrm{TE}_{01}$-mode switch cavity (7) connected to the waveguide by a diaphragm (6). The length of the waveguide section is chosen to ensure that the $\mathrm{TE}_{01}$ modes reflected from the conical taper and from the cavity sum in phase opposition when the cavity is in resonance. As a result, the total coefficient of $\mathrm{TE}_{02}$ $\rightarrow \mathrm{TE}_{01}$ mode conversion in the switch is $\sim 2-3 \%$. The switch cavity is formed by the diaphragm, a section of cylindrical waveguide, and the anode plate (9), which has an azimuthal slit that is interrupted by four small support sections. The slit is designed to allow an electron beam to enter the resonator at the field maximum of the $\mathrm{TE}_{01}$ mode excited in the switch cavity, and its 
Final Report on Phase II DoE SBIR grant DE-FG02-08 ER85206

Topic 50b: RADIO FREQUENCY POWER FOR LINEAR ACCELERATORS

\section{HIGH-POWER MICROWAVE SWITCH EMPLOYING ELECTRON BEAM TRIGGERING}

width and depth were chosen to limit the leakage of the cavity rf fields through the anode plate, in order not to degrade the natural Q-factor of the switch resonator. As a result, the intrinsic Q of the switch cavity remains high, and its total Q is determined principally by the coupling diaphragm, indicating that the cavity will be strongly over-coupled.

The amplitude of the electric field in the storage cavity and in the switch cavity in the regime of energy storage was calculated using the FDTD method [7]. The calculations showed that a storage cavity quality factor $Q_{L} \geq 10^{4}$ could be achieved when the $\mathrm{Q}$ of the switch cavity is $Q_{S} \approx 200-500$. The measured loaded $\mathrm{Q}$ of the storage cavities of the two compressor channels was $Q_{L 1} \approx Q_{L 2} \approx(2-3) \times 10^{4}$. The phase of the signal reflected from the switch cavity depends significantly on whether the cavity is in resonance or out of resonance [8]. When the switch cavity is changed from in resonance to out of resonance, the phase of the signal reflected from the cavity can change by $\sim 180^{\circ}$, causing the $\mathrm{TE}_{01}$ mode waves reflected from conical taper and from the switch cavity to begin to combine in phase. In this case, the $\mathrm{TE}_{02} \rightarrow \mathrm{TE}_{01}$ conversion coefficient can be increased up to $\sim 100 \%$, depending on the slope of taper (4). As a result, the microwave energy stored in the compressor cavity in the $\mathrm{TE}_{02}$ mode will be emitted from it in the $\mathrm{TE}_{01}$ mode, and the resonator can empty in one round-trip time of the power circulating in the $\mathrm{TE}_{02}$ mode.

The operation of the switch was modeled by studying the interaction of an electron beam injected into the fields of the $\mathrm{TE}_{012}$ mode excited in the switch resonator. The dynamics of the electron distribution in the switch were calculated by the Particle-in-Cell (PIC) method. In the calculations, the potential difference between the cathode and the anode was assumed to be $100 \mathrm{kV}$. The distance between the anode and the cathode and the beam current were varied during the calculations. The calculated distribution of the injected electrons in the resonator at a beam current of $200 \mathrm{~A}$ is shown in Fig. 1(b). The calculations showed that from $80 \%$ to $100 \%$ of

the electrons emitted by the cathode can be injected into the switch cavity, depending on the anode-to-cathode distance and the width of the cathode edge. 
Final Report on Phase II DoE SBIR grant DE-FG02-08 ER85206

Topic 50b: RADIO FREQUENCY POWER FOR LINEAR ACCELERATORS

HIGH-POWER MICROWAVE SWITCH EMPLOYING ELECTRON BEAM TRIGGERING

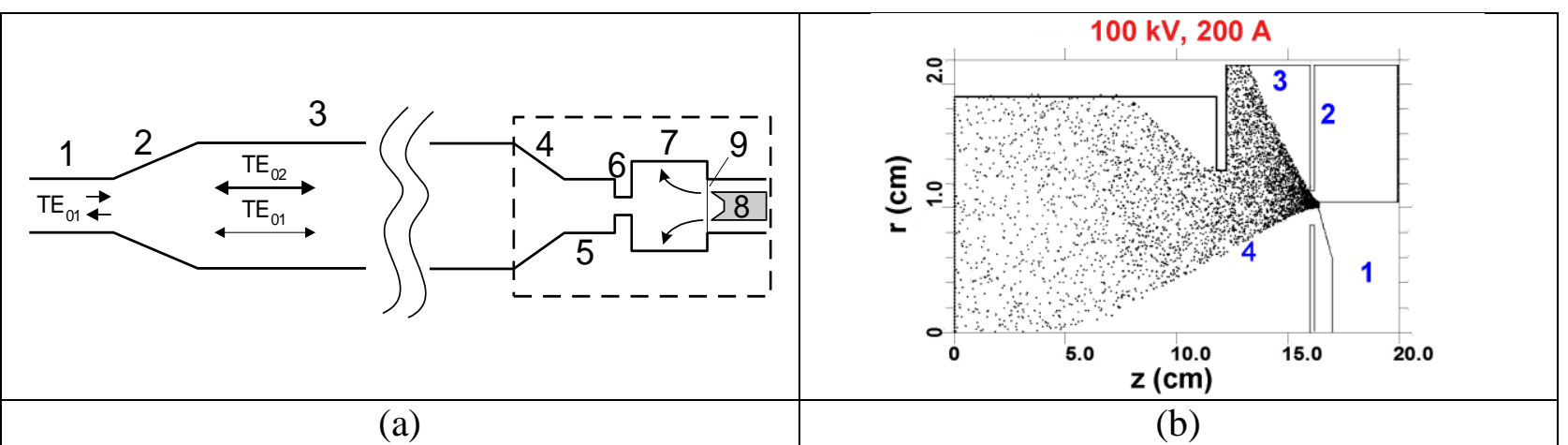

Fig.1. Schematic diagram of the single-channel compressor: 1 - input $\mathrm{TE}_{01}$ mode waveguide, 2 - taper, 3 - cylindrical waveguide cavity, 4 - conical taper, 5 - waveguide section, 6 - diaphragm, 7 - $\mathrm{TE}_{012}$-mode switch cavity, 8 - cathode, 9 - anode (output diaphragm); (b) Instantaneous calculated distribution of electrons (10) inside the switch resonator (7) at beam current 200A and anode voltage $100 \mathrm{kV}$.

The calculations also determined the phase and the reflection coefficient $R$ of the electromagnetic wave that was reflected from the switch resonator following injection of the electron beam. It was assumed that the switch cavity was driven externally beginning at $t=0$ to excite the $\mathrm{TE}_{012}$ mode at the resonant frequency $\mathrm{f}_{0}=11.43 \mathrm{GHz}$. The oscillations in the switch cavity were allowed to stabilized, and then an electron beam was injected into the cavity at $t^{*}=25 \mathrm{~ns}$. The dependence of the magnitude and phase of the reflection coefficient on the value of the electron beam current are shown in Fig. 3. The calculations were performed for two values of the beam current, $\mathrm{I}_{1}=200 \mathrm{~A}$ and $\mathrm{I}_{2}=400 \mathrm{~A}$.

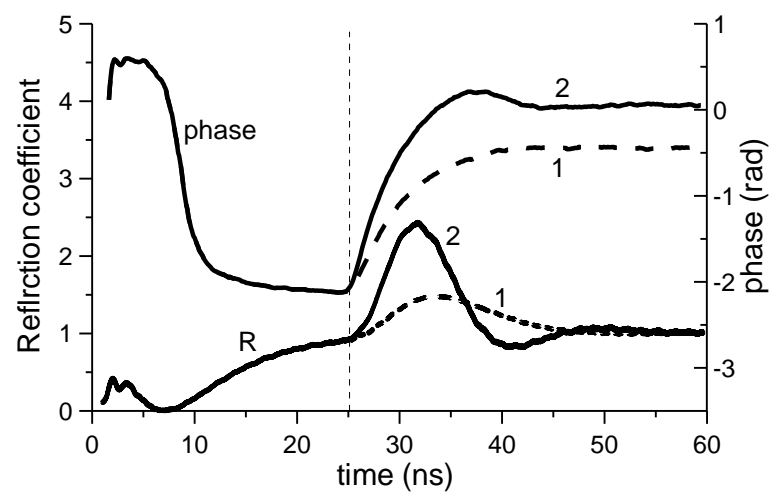

Fig. 3. Dependence of the magnitude and phase of the reflection coefficient $\mathrm{R}$ on time at different electron beam current: $1-\mathrm{I}_{1}=200 \mathrm{~A}, 2-\mathrm{I}_{2}=400 \mathrm{~A}$. 
Final Report on Phase II DoE SBIR grant DE-FG02-08 ER85206

Topic 50b: RADIO FREQUENCY POWER FOR LINEAR ACCELERATORS

\section{HIGH-POWER MICROWAVE SWITCH EMPLOYING ELECTRON BEAM TRIGGERING}

Fig. 3 demonstrates that the injection of the beam current beginning at $t^{*}=25 \mathrm{~ns}$ results in a rapid change in the phase of the reflected wave and also in the discharge of the energy stored in the switch resonator $(R>1)$. Because the cavity is over-coupled, the reflection coefficient is large both in resonance and out of resonance. It is evident that the larger beam current leads to a faster change in the phase and a larger total phase shift. The phase change time, $\tau_{\mathrm{p}}$, is determined by the time to stabilize the oscillation in the switch cavity, and is given by $\tau_{\mathrm{p}} \sim Q s / 2 \pi f_{0}$. For example, when the switch cavity Q-factor is $Q_{S}=200$, the characteristic phase shift time is $\tau_{p} \approx 3$ ns.

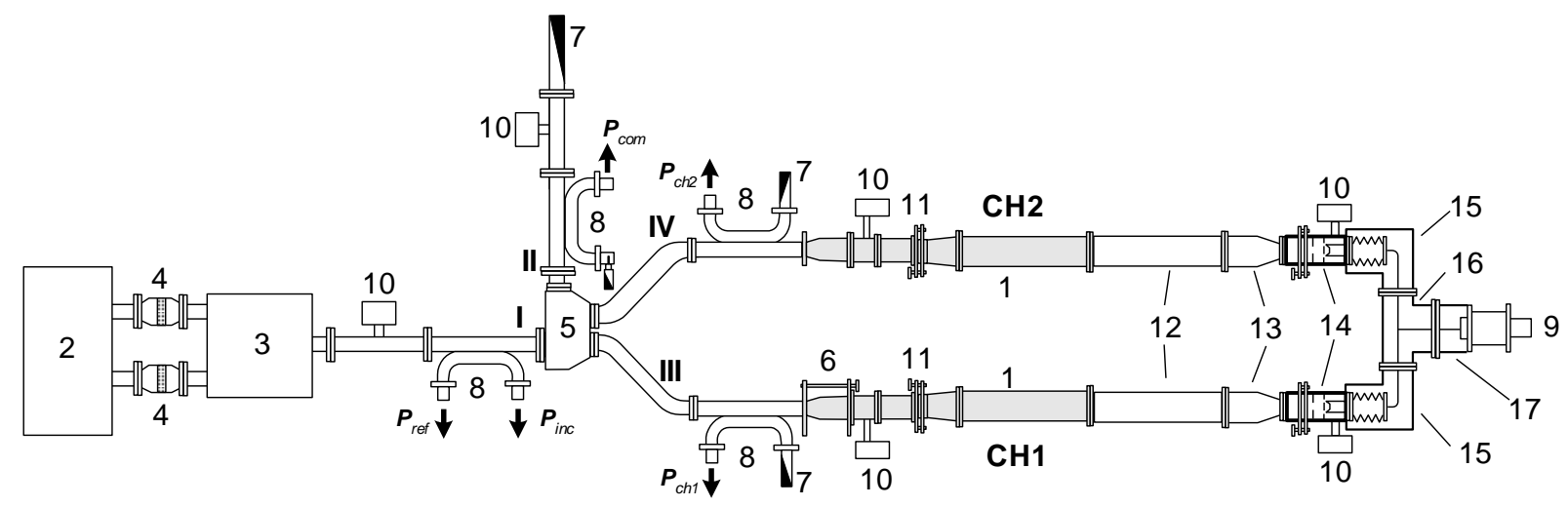

Fig.4. Schematic diagram of the experimental setup for tests of the two-channel microwave compressor with switches employing electron beam triggering: (1) single-channel of the compressor; (2) magnicon; (3) power combiner; (4) output window; (5) 3-dB hybrid coupler; (6) phase shifter; (7) matched load; (8) 55.5-dB directional coupler; (9) pulser; (10) ion pump; (11) mode converter, (12) cylindrical waveguide, (13) taper, (14) switch, (15), (16), (17) metal screen.

Fig. 4 shows a schematic diagram of the two-channel active pulse compressor that was studied using the magnicon as a high power $11.43 \mathrm{GHz}$ microwave source. Each energy storage cavity was formed by a conical taper, a cylindrical waveguide $210 \mathrm{~cm}$ long and $80 \mathrm{~mm}$ in diameter, and the switch that has been described above. The cylindrical waveguide included a section whose length could be mechanically adjusted to tune the cavity into resonance. A singlemode 3-dB hybrid coupler (5) was used to decouple the magnicon (2) from the reflected signal. The microwave power fed into branch $I$ of the coupler in the $\mathrm{TE}_{10}$ mode of rectangular waveguide is divided equally into branches III and IV. Then this mode is converted to the $\mathrm{TE}_{01}$ mode of circular waveguide by mode converters (11), and sent to the input of each channel. In 
Final Report on Phase II DoE SBIR grant DE-FG02-08 ER85206

Topic 50b: RADIO FREQUENCY POWER FOR LINEAR ACCELERATORS

\section{HIGH-POWER MICROWAVE SWITCH EMPLOYING ELECTRON BEAM TRIGGERING}

the energy storage regime, the power reflected from the resonators back into branches III and IV adds in phase in the output branch II, and cancel in branch I, so that no reflected microwave power returns to the magnicon. Phase shifter (6) is used to adjust the relative phase of the two channels to ensure cancellation of the reflected signal in branch I. Similarly, the output pulse from the two channels will add in phase in branch II and cancel in branch I.

Directional couplers $(55.5 \mathrm{~dB})$ were installed in each channel of the compressor. They provided a means to precisely tune the channels to the operating frequency and also enabled separate measurement of the compressed pulse in each of the channels. A high power matched load to receive the output pulse was placed at the end of branch IV. Signals proportional to $P_{i n c}$, the power incident on the compressor, $\mathrm{P}_{r e f}$, the power reflected back towards the magnicon, and $P_{c o m}$, the power sent forward to the load were measured by detectors via directional couplers (8) in branches I and II. The compressor vacuum was maintained at (3-5) $\times 10^{-7}$ Torr by means of ion pumps (10). The compressor was excited by the magnicon at power levels in the range of 1-9 MW and pulse durations $\tau=1-1.4 \mu \mathrm{s}$. The high level of microwave drive power was achieved by summing the power of the two magnicon output waveguides using combiner (3), which is based on a separate 3 -dB hybrid coupler.

The compressor was switched from the regime of microwave energy storage into the regime of energy extraction by means of electrically controlled switches employing electron beam triggering. A single pulser (9) was used to generate a $-100 \mathrm{kV}, 100 \mathrm{~ns}$ pulse that was fed to the cathodes in both channels of the compressor. The timing of the high voltage pulse was synchronized with respect to the magnicon pulse by time delay generators.

At the start of the experiment, each of the compressor channels was tuned to the magnicon frequency by adjusting the length of the corresponding storage resonator. With optimal tuning, the fraction of the incident pulse energy stored in the cavities reached 60. Next, the switch of each channel was separately tuned to maximize $\mathrm{P}_{\text {com }} / \mathrm{P}_{\text {inc }}$. Finally, the total power in the compressed pulse was measured with both channels operating simultaneously. When a high-voltage pulse was sent to the cathodes, an electron beam was injected into the switch cavities. This resulted in a change in the resonant frequency of the cavities, shifting them out of resonance, and, correspondingly, causing a change in the phase of the signal reflected from the switch cavities. As a result, the $\mathrm{TE}_{01}$ mode waves reflected from the conical taper and the switch 
Final Report on Phase II DoE SBIR grant DE-FG02-08 ER85206

Topic 50b: RADIO FREQUENCY POWER FOR LINEAR ACCELERATORS

\section{HIGH-POWER MICROWAVE SWITCH EMPLOYING ELECTRON BEAM TRIGGERING}

cavity began to add in phase and the $\mathrm{TE}_{02} \rightarrow \mathrm{TE}_{01}$ conversion coefficient abruptly increased. This caused microwave energy stored in the compressor at the $\mathrm{TE}_{02}$ mode to be emitted through the input horn in the $\mathrm{TE}_{01}$ mode. For these experiments, a conical taper with a calculated $\mathrm{TE}_{02} \rightarrow \mathrm{TE}_{01}$ conversion coefficient equal to $100 \%$ was used. In this case, the output pulse duration is determined by the round-trip time of the $\mathrm{TE}_{02}$ mode through the energy storage cavities.

The electron beam was produced by applying a high voltage pulse to a cylindrical molybdenum cathode $20 \mathrm{~mm}$ in diameter, corresponding to the diameter of the annulus in the anode plate. The cathode had a $\sim 50 \mu \mathrm{m}$ knife edge to achieve sufficient field emission when the high voltage pulse was applied. Preliminary investigations [9] showed that the cathode emitted non-uniformly from only a few cathode spots. However, it was found that depositing a film of diamond and diamond-like carbon on the surface of a cathode significantly increased the number of emission centers and substantially improve the uniformity of electron emission, as shown in Fig. 5. These photos of the operation of the molybdenum cathodes were taken by substituting a wire mesh for the anode plate of the switch. The diamond films were deposited on the surface of the molybdenum cathode in a microwave CVD systems using a $\mathrm{Ar} / \mathrm{H} 2 / \mathrm{CH} 4 / \mathrm{N}_{2}$ gas mixture under controlled conditions [9]. In the experiments, the diamond-coated cathodes reproducibly produced the $200 \mathrm{~A}$ electron beams required for switching, using a $100 \mathrm{kV}, \sim 100 \mathrm{~ns}$ high-voltage pulse, with no evident sign of degradation from use.
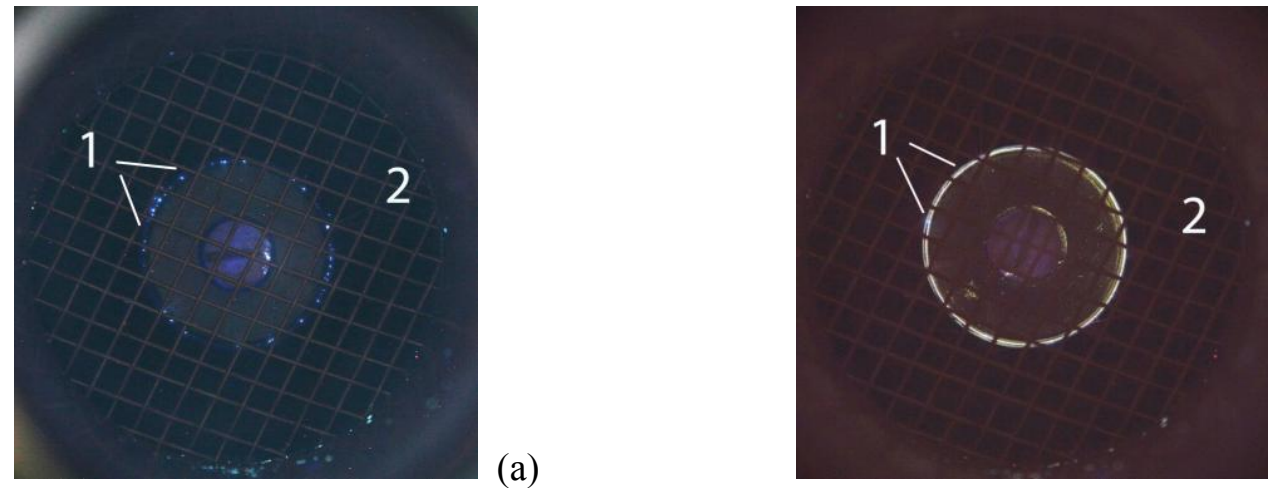

(b)

Fig.5. Photos of emission from a molybdenum cathode resulting from application of $100 \mathrm{kV}, 100 \mathrm{~ns}$ high voltage pulse (a) without and (b) with diamond coating on cathode: 1- cathode edge (behind wire mesh), 2- wire mesh. 
Final Report on Phase II DoE SBIR grant DE-FG02-08 ER85206

Topic 50b: RADIO FREQUENCY POWER FOR LINEAR ACCELERATORS

\section{HIGH-POWER MICROWAVE SWITCH EMPLOYING ELECTRON BEAM TRIGGERING}

Typical oscilloscope traces of the pulse compressor output are shown in Fig. 4. In Fig 4(a), the incident and compressed pulses are shown for $\mathrm{Q}_{\mathrm{s}}=400$ at an incident power of $\sim 9 \mathrm{MW}$. In this case, the compressed pulse has a peak power of $165 \mathrm{MW}$, a duration of $22 \mathrm{~ns}$ FWHM, a power gain of 18.3 , and an efficiency of $\sim 37 \%$. Power gains of up to 20 were observed at lower levels of incident power, indicating the ponderomotive effect of the rf fields of the switch cavity on the propagation of the electron beam, as shown in Fig.1(b). The total efficiency of compression, defined as the ratio of the energy in the compressed pulse to the energy in the incident pulse, was as high as 50\%. Also, the shape of the compressed pulses was stable and reproducible.
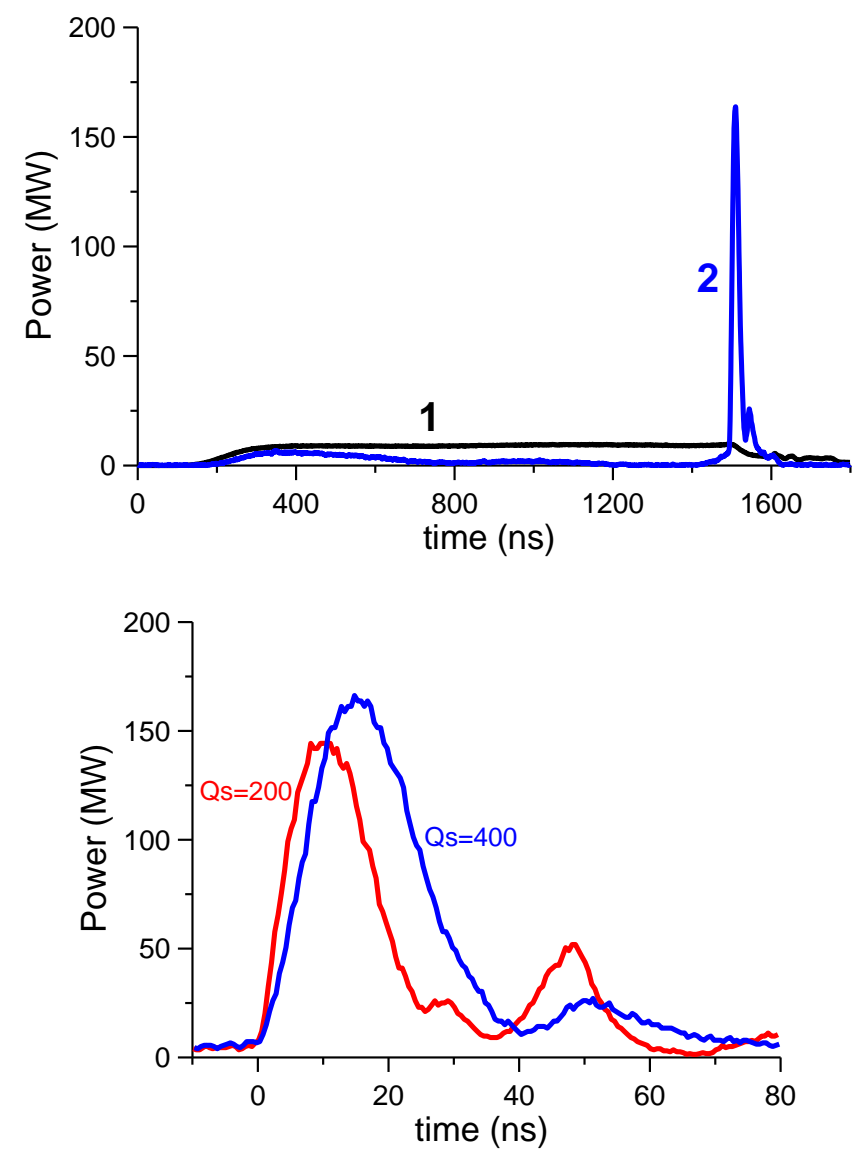

Fig.4. (top) Oscilloscope traces of the (1) incident and the (2) compressed pulses at $9 \mathrm{MW}$ incident power for $\mathrm{Q}_{\mathrm{s}}=400$; (bottom) comparison of the compressed pulses at $\mathrm{Q}_{\mathrm{s}}=200$ and $\mathrm{Q}_{\mathrm{s}}=400$. 
Final Report on Phase II DoE SBIR grant DE-FG02-08 ER85206

Topic 50b: RADIO FREQUENCY POWER FOR LINEAR ACCELERATORS

\section{HIGH-POWER MICROWAVE SWITCH EMPLOYING ELECTRON BEAM TRIGGERING}

The experiments showed that the parameters of the compressed pulse depended significantly on the parameters of the switch, including the distance between the anode and the cathode and the Q-factor of the switch cavity. These parameters were varied during the experiments to optimize the operation of the compressor. For example, as the distance between the anode and the cathode was decreased from $3.8 \mathrm{~mm}$ to $1.6 \mathrm{~mm}$, the stability of operation of the both channels increased and higher power gain was achieved. In one experiment, the Q-factor of the switch resonator was changed from $Q_{S}=400$ to $Q_{S}=200$ by reducing the resonator length from $38 \mathrm{~mm}$ to $19 \mathrm{~mm}$. As a result, the switch resonator was excited at the $\mathrm{TE}_{011}$ mode instead of the $\mathrm{TE}_{012}$ mode. The dependence of the parameters of the compressed pulse for these two different values of the switch Q-factor is shown in Fig. 4(b). It is seen that when the Q-factor of the switch cavity decreases, the rise time of the compressed pulse is reduced and the compressed pulse becomes more rectangular. However, the power multiplication factor also decreased to $16 \mathrm{x}$, indicating that the lower $\mathrm{Q}$ switch cavities required higher electron beam currents to cause a large enough shift in the resonant frequency for efficient switching to take place. Note that the secondary peak at $\sim 50 \mathrm{~ns}$ in both waveforms appears to be the result of a reflection from the output load at high peak powers due to rf breakdown in the load, and is not a result of energy remaining in the pulse compressor.

\section{CONCLUSIONS AND ACKNOWLEDGMENTS}

In conclusion, a two-channel active pulse compressor with switches based on a new concept of electron beam triggering was built and tested at high power levels at a frequency of $11.43 \mathrm{GHz}$. In experimental tests, the compressor produced $165 \mathrm{MW}$ in a $\sim 20$ ns pulse at $\sim 18 \mathrm{x}$ power gain and $\sim 140 \mathrm{MW}$ at $\sim 16 \mathrm{x}$ power gain in a $16 \mathrm{~ns}$ pulse with a $\sim 7 \mathrm{~ns}$ flat-top. In these tests, molybdenum blade cathodes with thin diamond coatings demonstrated good reproducible emission uniformity with a $100 \mathrm{kV}, 100 \mathrm{~ns}$ high voltage pulse. The new compressor does not

have the limitations of earlier types of active pulse compressors and can operate at significantly higher electric fields without breakdown. Also, it can be used to obtain rectangular output pulses. This suggests that a prototype active pulse compressor for a future $\mathrm{TeV}$ electron-positron collider 
Final Report on Phase II DoE SBIR grant DE-FG02-08 ER85206

Topic 50b: RADIO FREQUENCY POWER FOR LINEAR ACCELERATORS

\section{HIGH-POWER MICROWAVE SWITCH EMPLOYING ELECTRON BEAM TRIGGERING}

could be created by combining this type of switch with the longer resonant delay line of the SLAC pulse compression system [10].

This work was supported by the U.S. Department of Energy, Office of High Energy Physics, Grant No. DE-FG02-08ER 85206 and Interagency Agreement \#DE-AI02-01ER41170, and by the Russian Foundation for Basic Research, Grants No. 10-08-00260 and 12-08-00990.

\section{REFERENCES}

1. S.G. Tantawi, C.D. Nantista, V.A. Dolgashev, C. Pearson, J. Nelson, K. Jobe, J. Chan, K. Fant, J. Frisch, D. Atkinson, Phys. Rev. ST Accel. Beams 8, 042002 (2005).

2. A.L. Vikharev, A.M. Gorbachev, O.A. Ivanov, V.A. Isaev, S.V. Kuzikov, M.A. Lobaev, J.L. Hirshfield, S.H. Gold, and A.K. Kinkead, Phys. Rev. ST Accel. Beams 12, 062003 (2009).

3. O.A. Ivanov, V.A. Isaev, M.A. Lobaev, A.L. Vikharev and J.L. Hirshfield, Appl. Phys. Lett. 97, 031501 (2010).

4. O.A. Ivanov, V.A. Isaev, M.A. Lobaev, A.L. Vikharev and J.L. Hirshfield, Phys. Rev. ST Accel. Beams 14, 061301 (2011).

5. S.H. Gold, O.A. Nezhevenko, V.P. Yakovlev et al., in High Energy Density Microwaves, edited by R.M. Phillips, AIP Conference Proceedings 474 (AIP, New York, 1999), p. 179.

6. O.A. Nezhevenko, V.P. Yakovlev, S.H. Gold et al., Proceedings of the 2005 Particle Accelerator Conference, edited by C. Horak (IEEE, Piscataway, NJ, 2005), p. 1979.

7. A. Taflove, Advances in Computational Electrodynamics. The Finite-Difference TimeDomain Method (Artech, London, 1998).

8. A.B. Pippard, The Physics of Vibration, (Cambridge University Press, New York, 1989).

9. O. Ivanov, V. Isaev, D. Radishev, M. Lobaev, A. Vikharev, V. Chernov, A. Kozlov and J. Hirshfield, IEEE Trans. Plasma Sci. 39, 2794 (2011).

10. A.L. Vikharev, O.A. Ivanov, A.M. Gorbachev, M.A. Lobaev, V.A. Isaev, S.G. Tantawi, J.R. Lewandowski and J.L. Hirshfield, Phys. Rev. ST Accel. Beams Beams 14, 121302 (2011). 